\title{
ANATOMÍA Y ONTOGENIA DE SEMILLAS DE HELICTERES LHOTZKYANA (STERCULIACEAE)
}

\author{
por ANA MARÍA GONZÁLEZ1 y CARMEN L. CRISTÓBAL²
}

\begin{abstract}
Summary
The anatomy and ontogeny of the seeds of $H$. Lhotzkyana was studied, in order to know the structure and origin of its wing. Ripe seeds of $\mathrm{H}$. baruensis, representative of the genus, were also analized so as to compare both kind of seeds. The exotesta is made of longitudinally elongated sclereids, endotesta is parenchymatic with small patches of tannin cells. The principal mechanical layer of the seed coat is the exotegmen, formed by radially elongated macrosclereids; meso- and endotegmen are tanniniferous. The present study shows that the wing of the seeds of $H$. Lhotzkyana develops from the raphe, and consists of parenchymatic cells sourronding the rapheal vascular bundle and both sclerenchymatic epidermis.
\end{abstract}

\section{Introducción}

En el curso de la revisión taxonómica de Helicteres L. que está realizando la segunda de las autoras, llamaron la atención las semillas de unas pocas especies que se apartan de lo típico del género, por presentar una aleta a lo largo de la rafe y a veces otras más angostas en otras posiciones.

Una de las especies con esta particularidad es Helicteres Lhotzkyana (Schott \& Endl.) K. Schum., por lo que se han estudiado anatómicamente sus semillas, con el fin de conocer con precisiór: el origen y naturaleza de la mencionada expansión membranácea. Otras razones por las que se ha elegido esta especie son el buen desarrollo de la aleta y la disponibilidad de material. Las semillas características del género tienen rafe linear, como ejemplo se han analizado las semillas maduras de H. baruensis Jacq.

Rao (1954) describe la anatomía de la semilla de $H$. Isora L., especie del SE asiático y tipo del género. Corner (1976) estudia las semillas de H. sacarolha A. St.-Hil., A. Juss. \& Cambess.,

\footnotetext{
${ }^{1}$ Becaria de perfeccionamiento del CONICET

${ }^{2}$ Instituto de Botánica del Nordeste, UNNE-CONICET, CC: 209, Corrientes, Argentina.
}

del centro de Brasil y Bolivia. Ambas especies tienen semillas que responden al tipo más frecuente en el género.

Las especies aquí estudiadas se cuentan entre las de más amplia distribución en el género. El área de $H$. Lhotzkyana se extiende desde el SW de Bahía hasta la línea del trópico llegando al E de Bolivia y extremo NW de Argentina. $H$. baruensis se extiende desde el NW de México hasta el centro de Brasil.

Se han observado también semillas con aletas en $H$. carthagenensis Jacq., especie colombiana $y$, aunque menos notables, en $H$. semitriloba Bert. ex DC., ampliamente distribuida en el Caribe. Estas especies tienen también en común con $H$. Lhotzkyana las cápsulas rectas, es decir no espiraladas. Sin embargo no hay una correlación a nivel de género entre estos caracteres. No se han observado semillas con aletas en las especies del viejo mundo, donde todas excepto $H$. Isora tienen frutos rectos. En América hay también otras especies de frutos rectos con semillas donde la rafe es linear (Cristóbal, 1987).

\section{Material y Métodos}

Para el estudio ana tómico se utilizó material fijado en FAA (a) o seco y rehidratado (b) me- 
diante hervor en agua con unas gotas de detergente. Para la descripción morfológica externa se usaron ejemplares de herbario (c). Los ejemplares testigo están depositados en el Herbario del Instituto de Botánica del Nordeste (CTES) y son los siguientes:

Helicteres Lhotzkyana, (a) Paraguay, Dep. Amambay, Parque Nacional Cerro Corá, Ayo. Aceite, camino a Lorito Picada, Cristóbal y Krapovickas 2370. (b) Brasil, Mato Grosso, Jacuara, $25 \mathrm{Km}$ de Barra dos Bugres, camino a Cáceres, Krapovickas et al. 40213. (c) Brasil, Mato Grosso, Cuiabá, Coxipó da Ponte, Krapovickas y Cristóbal 43077.

Helicteres baruensis, (b) Brasil, Bahía, $18 \mathrm{Km}$ W de Bom Jesus da Lapa, Krapovickas 2999.2. (c) Brasil, Bahía, $5 \mathrm{Km} \mathrm{S}$ de Caetité, camino a Brejinho das Ametistas, Krapovickas 10077.

Para el análisis de la anatomía se confeccionaron preparados permanentes. Las semillas maduras se dejaron en etilendiamina (Carlquist, 1982) por períodos variables de acuerdo a su dureza antes de proceder a la deshidratación. La técnica usual de deshidratación con Alcohol Butílico Terciario (Johansen, 1940) se reemplazó por una serie de Deshidratante Histológico (Biopur (B) realizándose cinco cambios; el tiempo en cada paso varió de 15' para flores pequeñas a $30-45^{\prime}$ para semillas y frutos maduros; la preimpregnación se llevó a cabo con Aclarante-Preimpregnante (Biopur $\left.{ }^{\circledR}\right)$, dejándolo actuar primero 10 horas y luego de un cambio, 2 horas más. Estas soluciones se utilizan puras y pueden usarse varias veces.

Para la infiltración en parafina se utilizó la técnica de Johansen (1940) que consiste en colocar el material en una mezcla de partes iguales de alcohol butílico terciario y aceite de parafina en estufa a $60^{\circ}$ por $4-5$ horas y luego hacer dos cambios en parafina pura de 8-10 horas cada uno.

Se realizaron cortes seriados de $8-10 \mathrm{~mm}$ de espesor, tanto longitudinales como transversales, de flores y frutos en distintos estadios de desarrollo. Se colorearon con safranina - Astra blue (Luque et al. 1996).

Para la descripción del episperma se usó la terminología propuesta por Corner (1976).

\section{Resultados}

Los frutos de Helicteres son cilíndricos, ovoides hasta esféricos, espiralados o rectos, erectos o péndulos, leñosos, pentacarpelares, pentaloculares, pluriseminados. Las semillas se originan de óvulos anátropos, oblicuos a la placenta que es axilar y dispuestos en dos filas por carpelo.

La semillas, cuando completan el desarrollo quedan comprimidas dentro del lóculo, generalmente en una fila. Esto hace que se delimiten caras de contacto entre ellas y contra la pared del carpelo, por esta razón las semillas son desiguales entre sí. Cuando maduras, la superficie es verrucosa o rugosa y la rafe es más o menos sobresaliente. Finalmente quedan sueltas dentro del lóculo y caen por el extremo del fruto.

En el caso de H. Lhotzkyana los frutos son rectos y las semillas se mantienen en pares como los óvulos (Fig. 1 D). Esta disposición posiblemente se debe a que el fruto es recto y relativamente grueso, de hasta $3 \mathrm{~cm}$ de diámetro. Las semillas son irregularmente poliédricas, tienen las caras superior, inferior e interna (en contacto con la otra del par) casi planas y la cara externa convexa adaptándose a la pared del carpelo (Fig. 1 A). Miden aproximadamente $4 \mathrm{~mm}$ long. x $2 \mathrm{~mm}$ lat. $x 1,5 \mathrm{~mm}$ alt.,

Fig. 1. A-B: semillas de Helicteres Lhotzkyana, con la cálaza hacia arriba; A: par de semillas vistas desde el ápice del fruto, levemente inclinadas hacia afuera para mostrar la cara interna más o menos plana; B: semilla izquierda del par de la fig. A inclinada hacia adentro, mostrando la cara externa convexa, la superior plana y la aleta; C: semilla de H. baruensis, 3 / 4 lateral mostrando la rafe; D-L: H. Lhotzkyana; D: corte transversal del carpelo con un par de óvulos jóvenes; E: óvulo joven; F: óvulo antes de la fecundación; G: óvulo después de la fecundación; H: corte paradermal de las macrosclereidas del exotegmen; I: corte óptico de las esclereidas de la exotesta; J-L: cortes transversales del episperma; J: después de formado el micrópilo; K: inicio de la diferenciación del exotegmen; L: episperma completamente desarrollado. Abreviaturas: dg: endotegmen, dt: endotesta, eg: exotegmen, et: exotesta, g: tegmen, ob: obturador, sg: mesotegmen, t: testa, ta: células taníferas, te: tegumento externo, ti: tegumento interno. 

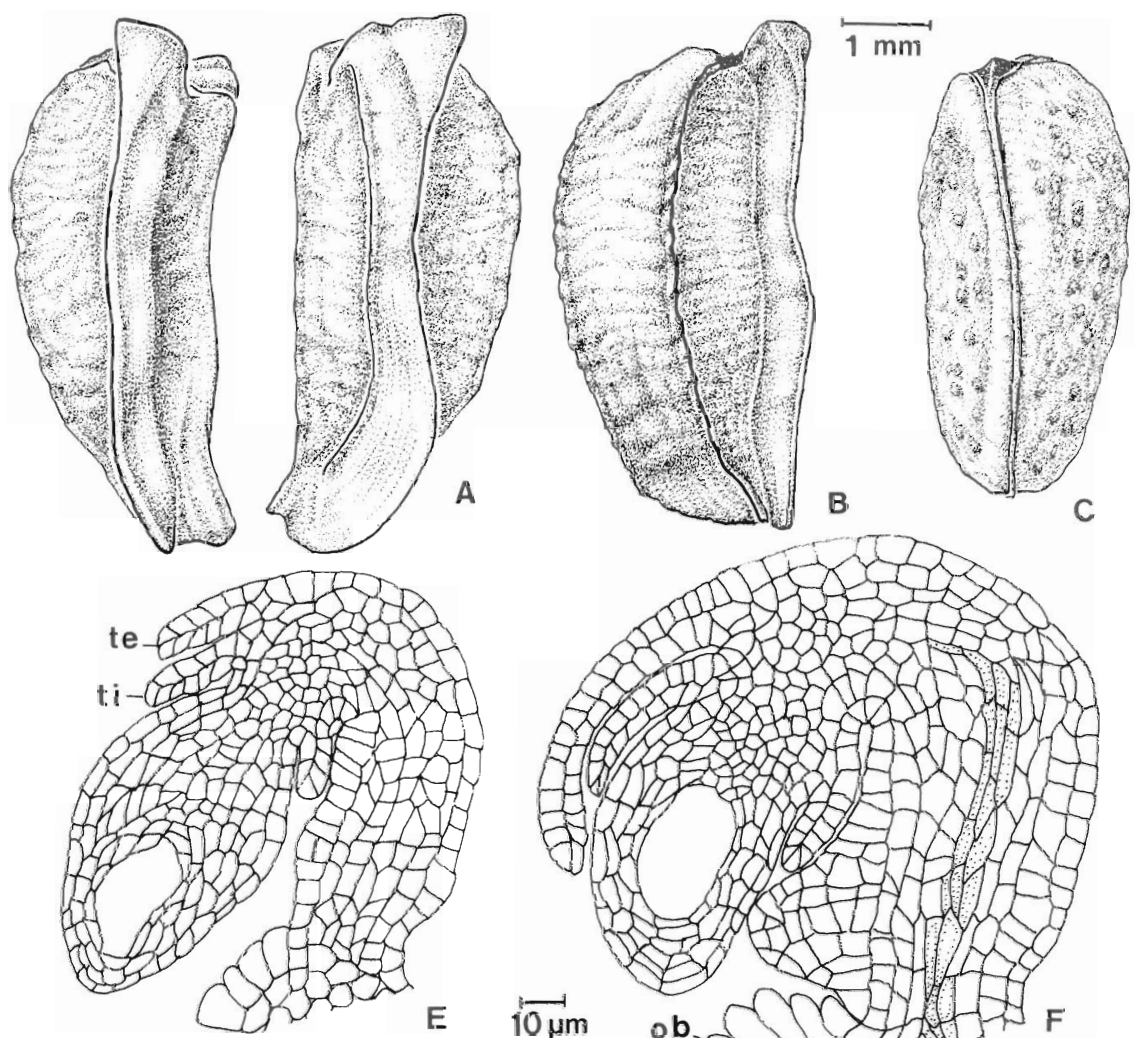

E $\longdiv { 1 0 \mu \mathrm { m } }$
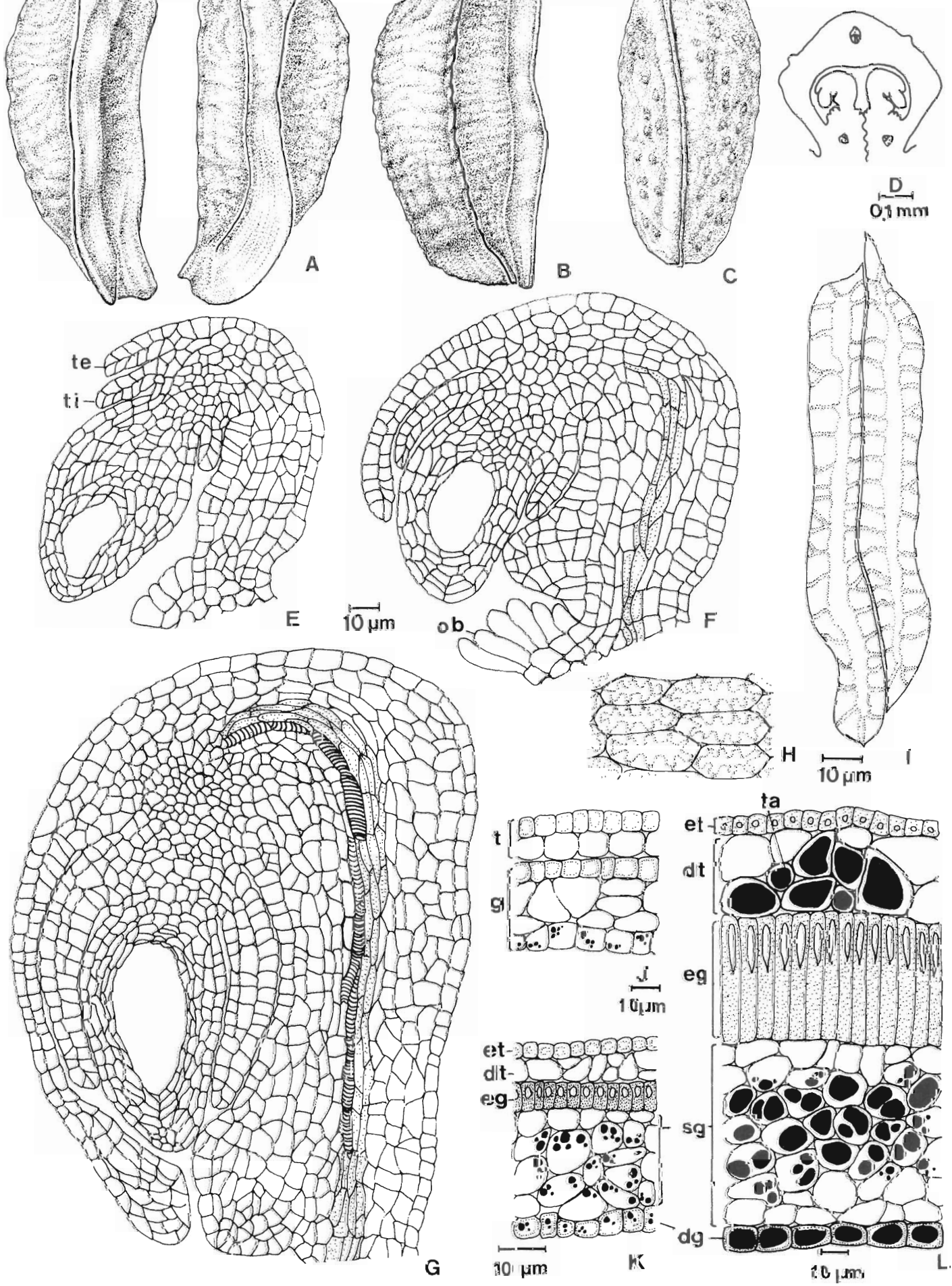

c
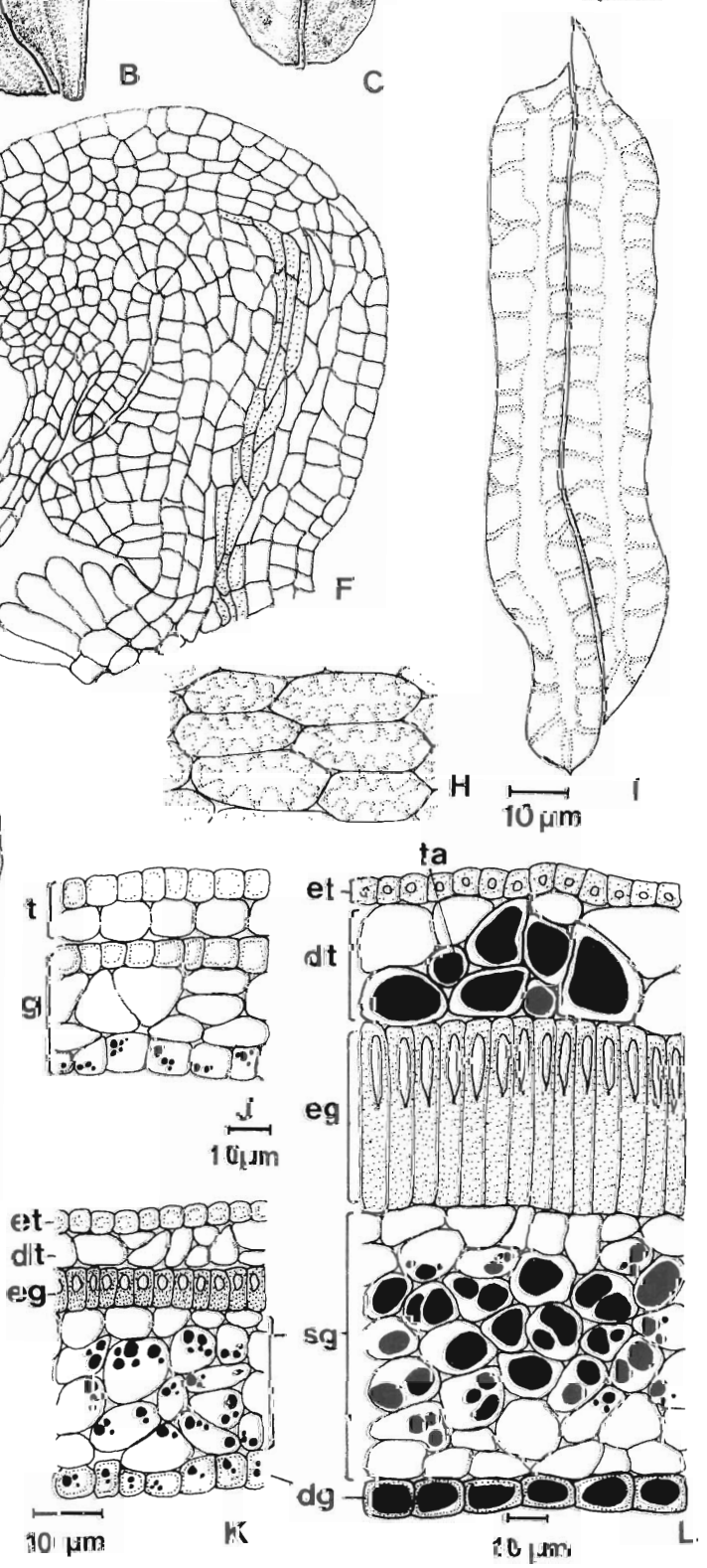
sin considerar la aleta. En la semilla madura, parte de la testa de las caras se separa como una película rugosa y al desprenderse deja a la vista verrugas de color castaño oscuro.

A lo largo de la rafe, las semillas tienen una aleta de 0,5-1 mm lat., que se dispone generalmente en la arista interna de la cara superior, desde el micrópilo hasta la cálaza. El haz vascular recorre la aleta en su parte media. La aleta es membranácea, nacarado-brillante y frágil, por lo que en la semilla madura puede desprenderse.

En algunas semillas a lo largo de las aristas de la cara inferior, se pueden desarrollar aletas mucho más angostas (Fig. 1 B). Las dos semillas de un par no están exactamente en el mismo nivel, sino una levemente más abajo que la otra, ni las filas están totalmente alineadas, por lo que a veces entre las dos semillas de un par puede asomar la aleta de una de las que están debajo.

En uno o en ambos extremos el fruto se afina por lo que el lóculo es más estrecho y sólo queda una semilla del par, que tiene forma cónica en el ápice del fruto y obcónica en la base. Por la misma causa otras pueden tener forma de tonel.

H. baruensis tiene el fruto espiralado, de 1$1,8 \mathrm{~cm}$ diám. Las semillas dispuestas en una sola fila, son irregularmente poliédricas, con las caras superior e inferior planas y caras laterales convexas, de $3 \mathrm{~mm}$ long. x 1,5 mm lat. $x 1 \mathrm{~mm}$ alt., verrucosas, castañas, con la rafe linear y la cálaza destacándose como una mácula negruzca (Fig. 1 C).

\section{Helicteres Lhotzky'ana}

Desarrollo de los óvulos: los primordios ovulares se inician como prominencias con una sola capa dérmica o dermatógeno, que cubre una masa central de células. Las células del dermatógeno se dividen activamente en su mayoría en el plano anticlinal, en tanto que las de la masa central lo hacen con distintas orientaciones.

La nucela adquiere considerable tamaño y prácticamente ha completado su giro de $180^{\circ}$ al iniciarse la diferenciación de los tegumentos (Fig. 1 E). Ambos tegumentos se originan por divisiones del dermatógeno; la porción del tegumento externo ubicada entre rafe y tegumento interno se origina a partir de la capa dérmica de la rafe. Las rafes de cada par de óvulos quedan en el plano medio del carpelo, en tanto que las nucelas se ubican hacia afuera (Fig. 1 D).

Antes de la fecundación el tegumento externo del óvulo cubre un poco más de la mitad de la nucela, en tanto que el tegumento interno llega a cubrir apenas una tercera parte de la misma; ambos tegumentos son biestratificados. El ápice nucelar queda casi en contacto con el obturador, formado por la epidermis papilosa de la placenta (Fig. 1 F, ob).

La cálaza es prominente, parenquimática; el haz vascular recorre la rafe hasta la cálaza donde se curva abruptamente.

En cada par de óvulos comienzan a superponerse levemente las respectivas rafes, esto se debe a que los óvulos deben inclinarse lateralmente para acomodarse al escaso lugar que tienen en el lóculo; así que en un corte transversal de ovario no se pueden ver la rafe y el saco embrionario al mismo tiempo. En corte longitudinal de ovario también se aprecia que los ôvulos están inclinados: funículo y ápice nucelar se orientan hacia la base del lóculo y la cálaza se ubica hacia el ápice.

Formación de las semillas: numerosos tubos polínicos llegan por el tejido de transmisión hasta el obturador y uno de ellos penetra por el ápice nucelar hasta el saco embrionario. Luego de producida la fecundación, el tegumento externo crece más rápidamente que el interno, formando el exóstoma antes que el tegumento interno alcance el ápice nucelar. El endóstoma se forma posteriormente.

Las células del obturador se reabsorben gradualmente. El haz de la rafe es colateral y con engrosamientos helicados en el xilema, al llegar a la cálaza se curva pero no se ramifica. En los primeros estadios del desarrollo, hay un gran aumento de masa celular, principalmente en el parénquima de la cálaza, rafe, exóstoma y nucela (Fig. $1 \mathrm{G}$ ).

Después de formado el micrópilo, el tegumento externo del óvulo forma la testa, posee dos capas celulares (Fig. 1 J, t), es más grueso en el exóstoma con 3-5 capas. El tegumento interno o tegmen consta de dos capas dérmicas y un estrato central de 1-3 capas celulares (Fig, $1 \mathrm{~J}, \mathrm{~g}$ ). 

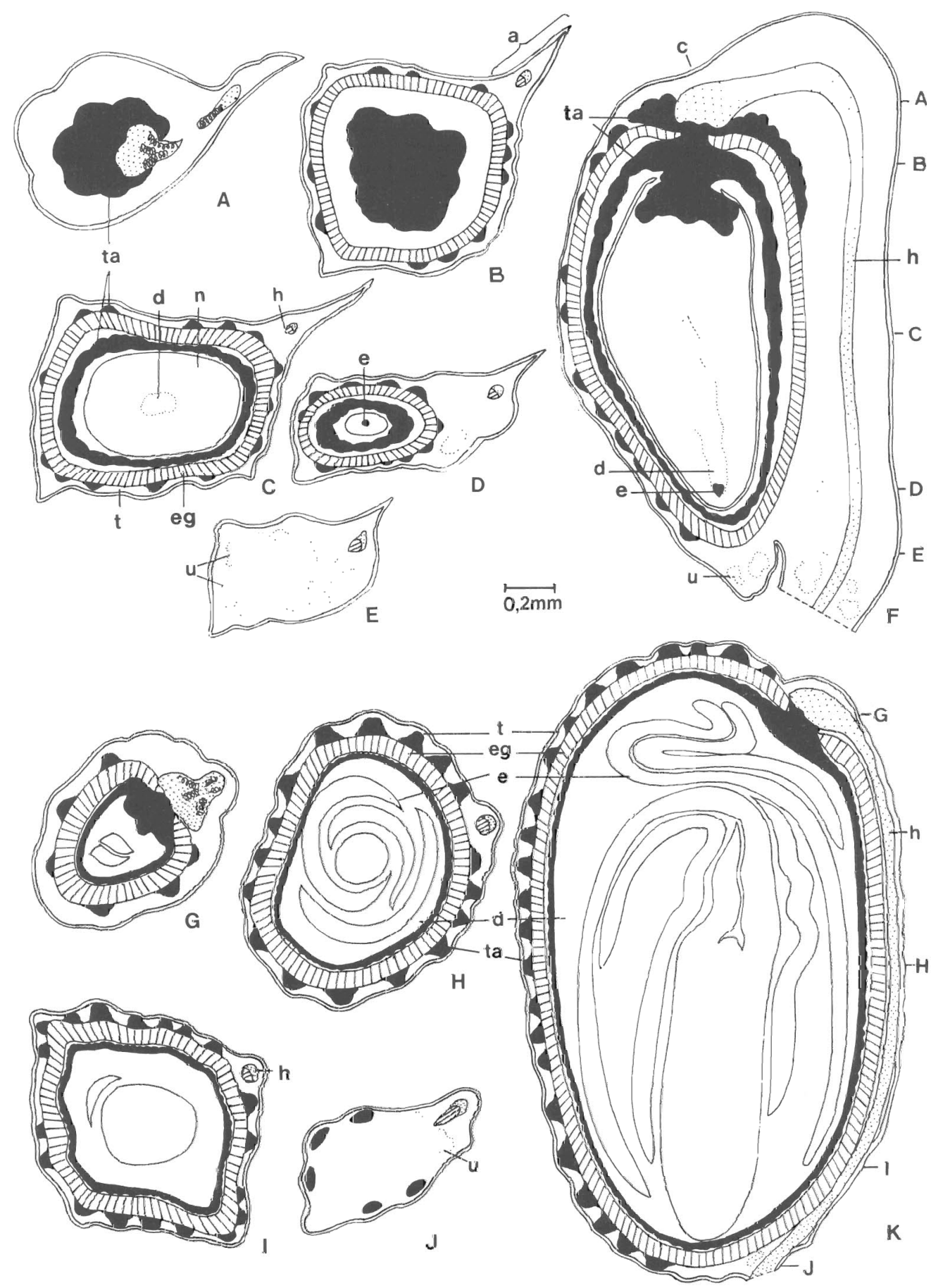

Fig. 2. A-F: semilla de Helicteres Lhotzkyana; A-E: cortes transversales seriados de semilla por los niveles indicados en F; F: corte longitudinal de semilla con episperma diferenciado y la rafe a la derecha; G-K: H. baruensis; G-J: transcortes por distintos niveles marcados en K; K: corte longitudinal de una semilla diferenciada. Abreviaturas: a: aleta, c: cálaza, d: endosperma, e: embrión, eg: exotegmen, h: hacecillo de la rafe, n: nucela, t: testa, ta: células taníferas, u: cavidades de mucílago. 
A medida que se desarrolla la testa, las células de la epidermis externa forman la exotesta, las células son alargadas y de sección cuadrangular (Fig. $1 \mathrm{~K}$, et). La epidermis interna está formada por células de tipo parenquimático constituyendo la endotesta, la cual incrementa gradualmente el número de capas (Fig. $1 \mathrm{~K}, \mathrm{dt}$ ). En el exóstoma y en la porción de la rafe adyacente a éste se diferencian cavidades lisígenas de mucílago (Fig. 2 E, F, u).

El tegmen posee 6-8 capas incluyendo ambas epidermis; la externa o exotegmen incrementa el número de divisiones anticlinales iniciando la diferenciación de una capa en empalizada (Fig. $1 \mathrm{~K}$, eg), en tanto que las células intermedias o mesotegmen y las de la epidermis interna o endotegmen acumulan sustancias tánicas. El endotegmen posee paredes celulares ligeramente engrosadas (Fig. $1 \mathrm{~K}$, sg, dg).

En estadios avanzados, las células alargadas de la exotesta se diferencian en esclereidas, dispuestas longitudinalmente en la semilla, miden 80-140 x 10-16 $\mu \mathrm{m}$ y tienen los extremos aguzados e imbricados, poseen pared secundaria muy gruesa, lignificada, con puntuaciones simples y escasamente ramificadas, el lumen es estrecho y puede llegar a obliterarse (Fig. 1 I, L); en la cálaza las esclereidas están dispuestas radialmente.

La endotesta está formada por un número variable de capas, desde un estrato en algunas zonas de las caras de la semilla hasta 10-12 capas en las aristas, son células parenquimáticas, aproximadamente isodiamétricas y de paredes delgadas; entre éstas hay grupos aislados de células taníferas (Fig. 1 L, dt, ta). La rafe adquiere gran desarrollo durante el crecimiento de la semilla, el haz vascular está rodeado por abundante tejido parenquimático (Fig. $2 \mathrm{~B}$, a; C, F, h).

Una capa de macrosclereidas forma el exotegmen, miden aprox. 50-70 x 6-9 x 17-25 $\mu \mathrm{m}$; la pared de la porción interna de estas células está notablemente lignificada, el lumen se observa estrellado en transcorte (Fig. $1 \mathrm{H}$ ) y queda reducido a la porción externa de la célula (Fig. $1 \mathrm{~L}$, eg); este estrato se dispone radialmente en la semilla y sólo está interrumpido en la cálaza (Fig. 2 F).

El mesotegmen está formado por 6-10 capas de células parenquimáticas, la mayoría son taníferas; en la región adyacente a la cálaza, todas las células son taníferas y se cuentan hasta 20 capas celulares. El endotegmen es completamente tanífero, de células cuadrangulares con pared ligeramente más gruesa (Fig. 1 L, sg, dg). En la semilla madura, el mesotegmen, la cálaza y la porción basal de los tegumentos son taníferos.

En la semilla que ha completado el desarrollo, el parénquima que rodea el haz de la rafe se comprime formando la aleta de la semilla. La aleta cstá constituida entonces por ambas epidermis, el hacecillo y restos de parénquima. En las aristas restantes el parénquima de la endotesta también es pluriestratificado, por lo que pueden formarse aletas más angostas (Fig. 2 B-D). En cortes transversales seriados de semilla se puede observar la posición de las aristas y de la rafe que forma la aleta de la semilla (Fig. 2 A-E, a).

El parénquima no tanífero de la endotesta termina por romperse, y se separa junto con las esclereidas de la exotesta, esta película finalmente se desprende y se observan los grupos de células taníferas como verrugas oscuras.

La forma final de la semilla depende de la posición que ocupa luego de la fecundación. Por esta razón, la aleta de la rafe puede quedar en una de las aristas internas o presentarse ocasionalmente en una de las caras.

La cálaza presenta una depresión circular alrededor de la terminación del haz vascular, esta depresión se forma por la deshidratación del parénquima, lo que pone en evidencia el reborde de las macrosclereidas del exotegmen, interrumpidas en la base del tegumento externo; a su vez las esclereidas de la epidermis acompañan esta disposición radial.

\section{Helicteres baruensis}

Anatomía de la cubierta seminal: la testa está formada por la exotesta, constituida por esclereidas dispuestas longitudinal y tangencialmente, con paredes muy gruesas, lignificadas, con puntuaciones simples y escasamente ramificadas y lumen reducido o prácticamente obliterado. La endotesta está formada por 3 a 5 capas de células parenquimáticas más o menos isodiamétricas con paredes celulares ligeramente engrosadas; hay grupos muy nu- 
merosos de células taníferas con paredes gruesas.

En el tegmen, el exotegmen está formado por macrosclereidas de aprox. $100 \times 10-12 \mu \mathrm{m}$, el lumen estrellado está reducido a la mitad externa de la célula; este estrato está interrumpido en la cálaza. El mesotegmen completamente tanífero y colapsado en la semilla madura, tiene de 5-8 capas en los lados de la semilla y de hasta 20 capas en la cálaza. El endotegmen tiene células de paredes ligeramente engrosadas, con depósitos tánicos.

La rafe se observa como un cordón sobresaliente (Fig. 1 C). En cortes transversales seriados se observa el haz vascular colateral, sin ramificaciones (Fig. $2 \mathrm{I}, \mathrm{K}, \mathrm{h}$ ). Las aristas están conformadas por ambos tegumentos (Fig. 2I).

Los abundantes grupos de células taníferas de la endotesta forman las verrugas, el parénquima incoloro ubicado entre ellos se deshidrata y se aplasta en la semilla seca, de modo que las esclereidas de la exotesta se adhieren y acomodan a la forma de las verrugas.

En la zona adyacente a la cálaza se interrumpe el estrato de macrosclereidas del exotegmen, externamente esto se aprecia como una depresión que rodea la terminación del haz vascular, las esclereidas de la epidermis acompañan esta disposición radiada. La coloración rojiza de la cálaza se debe a la presencia de células taníferas.

En ambas especies, el embrión completa su desarrollo recién cuando la semilla ha alcanzado su máximo tamaño. Los cotiledones son foliáceos, muy desarrollados y se encuentran plegados alrededor del eje hipocótiio-radícula. Es muy notable la abundancia de drusas en los cotiledones.

\section{Discusión y Conclusiones}

El desarrollo observado en los óvulos en $H$. Lhotzkyana concuerda con el descripto en $H$. Isora por Rao (1954). El obturador placentario está en contacto con el ápice de la nucela, por donde entra el tubo polínico. Los tegumentos del óvulo forman la micrópila después de la fecundación.

Luego de la fecundación la diferenciación de la semilla se produce principalmente en los tegumentos quedando el embrión en estado inmaduro hasta que la semilla alcanza su máximo tamaño, esta característica fue señalada por Corner (1976) en H. sacarolha.

La aleta de la semilla de $H$. Lhotzkyana es producto del desarrollo del parénquima de la rafe y de la epidermis. En la semilla madura la aleta con el hacecillo puede desprenderse y caer. Las otras aristas de la semilla también se forman por la proliferación del tejido parenquimático, pero en menor grado y dependen de la posición que tome la semilla dentro del lóculo.

H. baruensis también presenta aristas por la presión de las semillas dentro del lóculo, sin embargo en las aristas participan todos los estratos de ambos tegumentos seminales. El estrato parenquimático de la endotesta es bastante uniforme en todo el contorno de la semi1la, inclusive en la rafe, razón por la cual esta especie carece de aletas.

La parte exterior de la testa de H. Lhotzkyana es membranácea y nacarada, desprendiéndose con facilidad debido a la ruptura del parénquima de la endotesta sobre las verrugas de células taníferas, las que se observan al caerse la película externa, conformada por exotesta y parte de la endotesta. En H. baruensis la exotesta no se desprende ya que el parénquima incoloro de la endotesta es escaso y de paredes engrosadas, por lo que se deshidrata y aplasta pero no se rompe, de este modo las esclereidas de la exotesta se adhieren firmemente a las verrugas.

En las semillas de $H$. baruensis y $H$. Lhotzkyana la exotesta está formada por esclereidas longitudinales, las restantes características del episperma concuerdan básicamente con las observadas en $H$. Isora (Rao, 1954) y en $H$. sacarolha (Corner, 1976).

Se han encontrado semillas con aletas en tres especies americanas que tienen frutos rectos: H. semitriloba, $H$. carthagenensis y $H$. Lhotzkyana. Sin embargo estas características no siempre están ligadas. Hay otras especies americanas con frutos rectos y todas las asiáticas, excepto $H$. Isora, que tienen semillas sin aleta es decir con la rafe linear.

La aleta aparece en tres especies altamente diferenciadas, tanto que $H$. Lhotzkyana y $H$. carthagenensis han sido descriptas originariamente como géneros independientes. Es inte- 
resante la manifestación tan esporádica de este carácter; las áreas de distribución de estas especies se suceden sin superponerse y dos de ellas están respectivamente en los límites norte y sur del género en América.

\section{Bibliografía}

CORNER, E. J. H. 1976. The seeds of Dicotyledons. 2 Vol. Cambridge University Press.

CARLQUIST, S. 1982. The use of etilendiamine in softening hard plant structure for paraffin sections. Stain Techonology 57: 311-317.

CRISTOBAL, C. L. 1987. Un Helicteres nuevo de México, nexo entre las especies asiáticas y americanas. Bonplandia 6(i): 71-81

LUQUE, R.; SOUSA. H. C. \& KRAUS, J. E. 1996. Métodos de coloração de Roeser (1972) - modificado - e Kropp (1972) visando a substituição do azul de astra por azul de alcião 8 GS ou 8 GX. Acta bot. bras. 10(2): 199-212

JOHANSEN, D. A. 1940. Plant Microtechnique. McGraw Hill Book Co. New York. 511 págs.

RAO, C. V. 1954. Contributions to the embryology of Sterculiaceae. Indian Bot. Soc. J. 32: 208-238. 